\title{
A SAR Image-Based Tool for Prompt and Effective Earthquake Response
}

\author{
R. Guida and P.T.B. Brett \\ Surrey Space Centre \\ University of Surrey \\ Guildford, UK \\ r.guida@surrey.ac.uk
}

\begin{abstract}
Recently, a new concept for detection of damaged infrastructure after earthquakes has been introduced, based on analysis of double reflection lines in SAR images. This paper describes the development of a processing step for extraction of double-reflection lines, and its implementation. In particular, an unsupervised bright line detector working on the ratio of preand post-event single look complex SAR data is introduced, and is demonstrated using COSMO-SkyMed SAR data from the 2009 L'Aquila earthquake.
\end{abstract}

\section{INTRODUCTION}

Natural hazards all over the world have devastating effects. Recent earthquakes, in particular those in Italy, Haiti and China in 2009 and 2010, have demonstrated the human implications, including severe casualties and widespread homelessness, and also the long-term damage to infrastructure, which has had a shocking impact on local and regional economies. A 2008 report (thus excluding the former earthquakes) stated that in the last two decades earthquakes have accounted for the loss of well over half a million lives, and an economic cost of billions of dollars [1].

There is an urgent need for preparation to act in response to events such as these, as stressed by the Hyogo Framework, and proper investment in preparedness is certainly worthwhile.

Several projects have been set up to raise awareness of the issue and to support decision-making in the immediate aftermath of an earthquake event. For example, the PAGER [2] and the EXTREMUM projects [3] are working in this direction. On one hand, these projects address the requirement of real time information availability, but on the other they mainly produce maps of affected areas based on estimations but not real measurements. For example, mainly based on loss models, the PAGER project provides only estimates of the number of people and cities exposed to severe shaking following significant earthquakes based on the magnitude and location of the event and the application of groundmotion prediction equations. Consequently, the real damage to buildings (and not simply the estimate of it) cannot be assessed by the PAGER project. Similarly, the EXTREMUM project analyses spatial and descriptive data including seismic risk and estimate the vulnerability of structures. None of these various projects are based on measurements in real time from high resolution remote sensing data, and it is this lack that has prompted the authors' research, carried out at the University of Surrey.

A map of damaged areas based on measurements, rather than on estimations, can provide a better identification of severely hit infrastructure, and thus also indicate the areas where prompt intervention is more urgently required.

A new concept about how to produce this kind of map has been recently presented in literature [4], [5]. The underlying rationale is based on the exploitation of just one pair of high resolution Synthetic Aperture Radar (SAR) images, one before and one soon after the event, acquired in as similar conditions as possible with respect to radar parameters. The requirement of high resolution SAR is dictated by the scenario: urban areas present many features visible at or around metre resolution, and the reduction in coverage area resulting from the high resolution acquisition mode is outweighed by the benefits of having available a detailed representation of the area under study. The issue of temporal resolution is also a crucial one for monitoring earthquakes, but fortunately this is well addressed by the most recent generation SAR platforms and sensor networks.

Because the rationale strongly depends upon the accurate extraction of double reflection lines, the processing 'block' implementing this step has been further reviewed in this paper, and an alternative version is proposed. In Sec. II the new rationale is presented highlighting the differences from the previous version. The main improvement lies in the implementation of an unsupervised ridge detector, which is introduced and applied to SAR data in Sec. III.

\section{Tool RATionale}

In [4], [5] a new concept for a SAR-based tool mapping damaged areas in urban city centres was presented. A measurement of the damage incurred was possible thanks to the measurement of the change in the brightness of double reflection lines and the proper inversion of scattering models for canonical urban structures [6].

In that version of the tool, some steps were supervised or carried out manually, which imposed a particular structure for the full chain. For example, as shown in Fig. 1, the block for double reflection line extraction was designed to first map the double reflection lines on the pre-event image using an edge detection filter along with contextual information, and to then look for their corresponding parts in the ratio image (i.e. the 


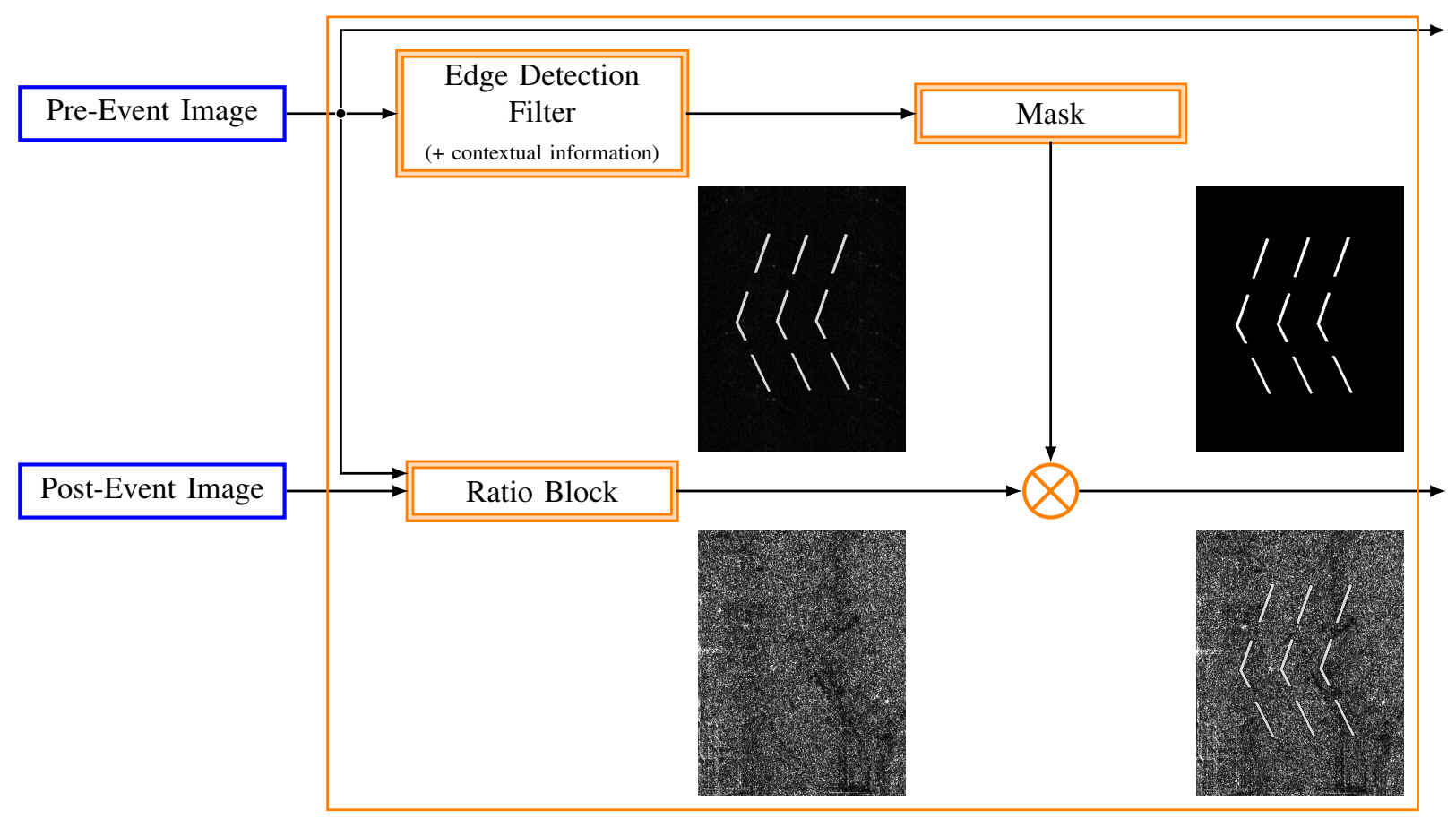

Fig. 1. Double reflection line extraction block using edge detection filter applied to pre-event SAR image.

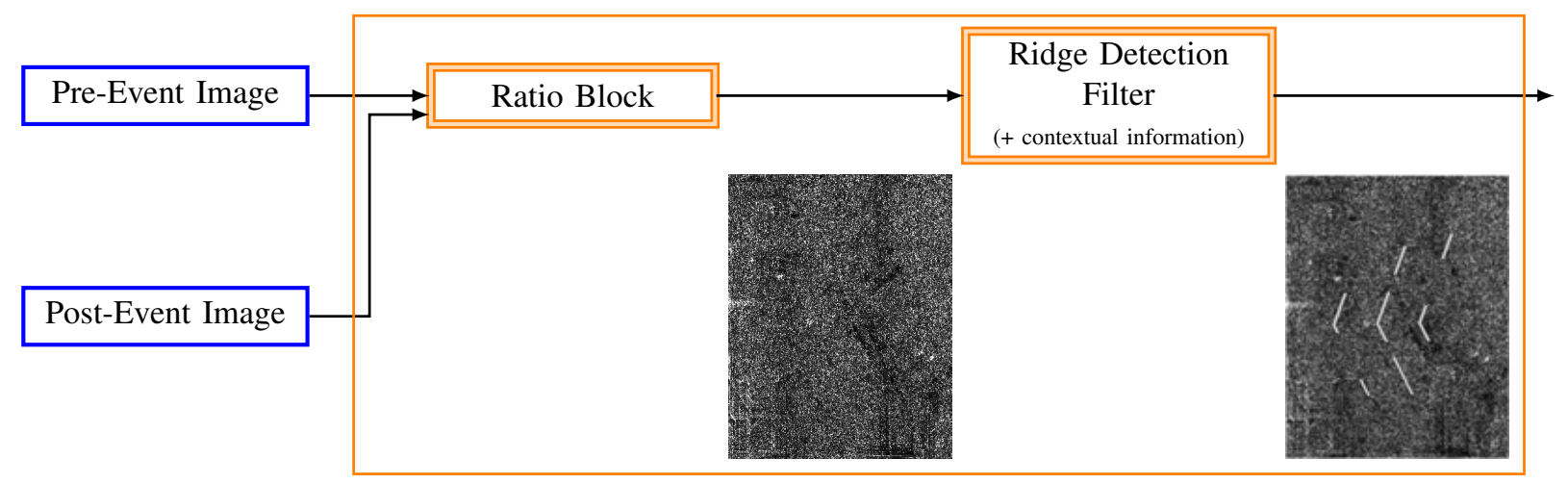

Fig. 2. Double reflection line extraction block using ridge detection filter applied to ratio SAR image.

line representing the ratio of the same double reflection line in the pre- and in the post-event SAR image). The choice of this structure was due to two reasons:

1) the main idea was to map all double reflection lines in order to check for changes to each of them;

2) direct extraction of double reflection lines in the ratio image was not possible because of the high noise in this kind of image (where the dynamics of speckle increases, side lobes may appear stronger and other effects due to processing are more likely to become visible).

The authors next addressed this second issue, and here propose a second possible version of their tool where the electromagnetic part is left unchanged.

It is well known that the extraction of double reflection lines is not only a problem of bright line extraction. Indeed, backscattering due to corner reflectors (as dihedrals formed be- tween a building wall and the ground) and specular reflections due to gabled roofs may both appear as bright narrow lines in SAR images but only the first ones are of primary interest for the authors. This is because, in the tools we are developing, the change detection block following the one for the extraction of double reflection lines fully exploits the electromagnetic modelling of this kind of contribution for a first estimation of the damage occurred [4], [5]. Nevertheless, at the moment the identification of bright lines is the first step in the detection change but it is accompanied by use of contextual information for distinguishing between the two kinds of scattering.

In the first version of the tool (Fig. 1), pre- and post-event SAR images of an urban area are input to a ratio block and an edge detection filter. The former simply computes the ratio of the pre- over the post-event single look complex SAR image after proper co-registration of the two images, for which 


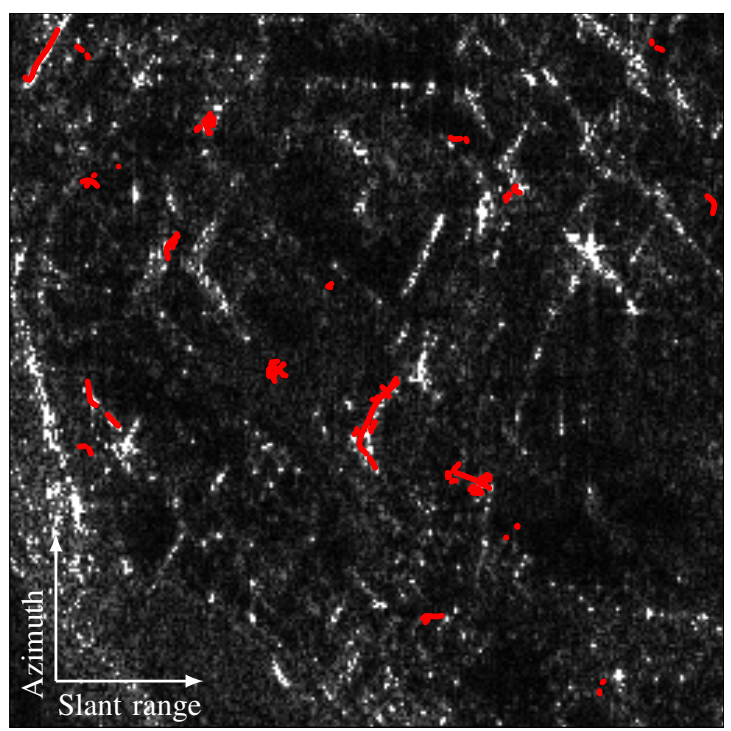

Fig. 3. Ratio image bright line detection applied to an area of L'Aquila, Italy. Processed from COSMO-SkyMed product (C) Agenzia Spaziale Italiana 2009. All rights reserved.

the assumptions are those made in [4], [5]. A mask is then produced and multiplied by the ratio image in order to have all the corresponding double reflection lines mapped on this.

In the second version, as shown in Fig. 2, the block of double reflection lines extraction is much simpler. The ratio is computed again and then a ridge detection filter is applied directly at the ratio image. The ridge detector that has been implemented employs a single-scale scale-space ridge detection method [7] based on the height definition for a ridge [8], followed by a threshold classification based on the $\gamma$ normalised square principal curvature difference ridge strength metric $\mathcal{N}_{\gamma-\text { norm }} L$ [7].

With this new version of the tool, obviously, not all the ratios of double reflection lines can be detected but only the strongest, i.e. those presenting a more severe damage. The reason for employing a version of the tool like this is to have a first quicker, even if coarser, detection of the most damaged areas/infrastructures. In a second pass, the previous version can be applied to obtain more detailed mapping. A modular and versatile tool is then imagined, with production of maps every time more and better defined.

\section{RESUlts}

To demonstrate the detector, it was applied to the same set of COSMO-SkyMed images before and after the April 2009 earthquake in L'Aquila, Italy, that were used in [4], [5]. Fig. 3 shows the results of applying the bright line detector to a $256 \times 256$ pixel SAR image cut, centred on an area of central L'Aquila, at a scale $t=8$ and with a ridge strength metric threshold $\mathcal{N}_{\gamma-\text { norm }} L>0.05$. The results have been superimposed on the prior image in order to show how they relate to bright lines that have been detected as absent.

The algorithm has successfully identified the major damage sites in the area (located at the centre, left, and extreme topleft of the figure) that were also detected in [4], [5]. However, from comparison of the map in Fig. 3 with an optical image of the area acquired after the event, we believe that false positives have also been detected arising from noise in the ratio image. Unfortunately, the ground truth for the processed images is not available. Consequently, the completeness and correctness of the new detector cannot be fully explored on this dataset. This problem will be overcome by the authors by the use of simulated SAR images of canonical scenes, and also by investigating recently-acquired SAR datasets covering the Haiti earthquake in 2010. These further results will be presented at the conference.

Compared with the previous version of the tool, the main advantage in this latest version is the removal of the manual ridge location step, making the detector fully unsupervised. As a consequence of this, the speed has been greatly improved. Indeed, the algorithm as currently implemented was run on a single core of an Intel Core i5 CPU at $2.67 \mathrm{GHz}$, and took 7.83 s to generate the image shown in Fig. 3. The same task, manually performed, took around 2 hours for the same SAR image cut.

With this improvement in time performance, the application of this approach to the full dataset is now feasible, and will be presented at the conference. These results will be numerically compared with mapping results deriving from the application of the previous detection chain.

\section{CONCLuSions}

An improved version of a SAR-based tool for mapping damaged infrastructure in urban areas has been presented. As its first version it relies upon a block for the extraction of double reflection lines which is still a delicate task. A new way to accomplish this task has been here suggested and, differently from the previous version, the new block directly applies to the prior/posterior ratio image and, above all it is unsupervised.

The drawback is represented by the false positives which are typical of such unsupervised approaches, but this is an active area of research, and the authors are currently investigating approaches based on statistical modelling of the ratio image in order to improve and quantify the performance.

Conversely, the simple and quick implementation can provide what is really necessary in the first hours after an earthquake: a real time map of affected areas. Moreover the tool in the new version is optimised for detecting severely damaged infrastructure, which is although being only a partial result, also represents the most urgent information.

\section{ACKNOWLEDGEMENT}

The authors would like to thank the Remote Sensing Group at the University of Naples Federico II and the Italian Space Agency for providing the COSMO-SkyMed SAR images used in this study. 


\section{REFERENCES}

[1] Natural catastrophes 2008: analyses, assessments, positions. Topics Geo. Munich Re Group, Munich, Germany, 2009.

[2] P.S. Earle, D.J. Wald, K.S. Jaiswal, T.I. Allen, K.D. Marano, A.J. Hotovec, M.G. Hearne, and J.M. Fee. Prompt Assessment of Global Earthquakes for Response (PAGER): A system for rapidly determining the impact of global earthquakes worldwide. U.S. Geological Survey, 2009. Open-File Report 2009-1131.

[3] N. Frolova, V. Larionov, S. Sushchev, and A. Ugarov. Extremum system for earthquake risk and loss assessment. In Conference Skopje Earthquake - 40 Years of European Earthquake Engineering, 2003.

[4] R. Guida, A. Iodice, and D. Riccio. An application of the deterministic feature extraction approach to COSMO-SkyMed data. In Synthetic Aperture Radar, 2010 European Conference on, pages 1014-1017, June 2010.

[5] R. Guida, A. Iodice, and D. Riccio. Monitoring of collapsed built-up areas with high resolution SAR images. In Geoscience and Remote Sensing Symposium, 2010. IGARSS 2010. IEEE International, July 2010. Forthcoming.

[6] G. Franceschetti, A. Iodice, and D. Riccio. A canonical problem in electromagnetic backscattering from buildings. Geoscience and Remote Sensing, IEEE Transactions on, 40(8):1787-1801, Aug. 2002.

[7] T. Lindeberg. Edge detection and ridge detection with automatic scale selection. International Journal of Computer Vision, 30(2):117-154, 1998.

[8] D. Eberly, R. Gardner, B. Morse, S. Pizer, and C. Scharlach. Ridges for image analysis. Journal of Mathematical Imaging and Vision, 4(4):353373, 1994. 